\title{
Suspected Anterior Horn Disease Mistaken as a Mass in the Sacral Plexus: A Case Report
}

\author{
Do-Hyung Kimª, b, Soo Joo Lee ${ }^{\mathrm{a}}$
}

\begin{abstract}
Anterior horn disease is a medical disorder affecting the anterior horn of the spinal cord that contains the motor neurons responsible for body muscles. Poliomyelitis, spinal muscular atrophy, amyotrophic lateral sclerosis and progressive muscular atrophy are diseases that frequently include problems within the anterior horn. Anterior horn disease is characterized by selective involvement of muscles. Here, we describe a young female patient with ankle weakness and selective muscle atrophy. Her initial pelvic magnetic resonance imaging revealed a suspected intraneural ganglion lesion affecting the first sacral nerve of the sacral nerve plexus. Eventually, though, she was suspected of anterior horn disease based on the clinical symptoms and electrophysiological findings after orthopedic surgical procedure.
\end{abstract}

Keywords: Anterior horn disease; Motor neuron injury; Limb weakness; Nerve conduction; Peripheral nerve; Spinal cord; Electromyography

\section{Introduction}

Spinal disorders can present with sensory symptoms, motor symptoms, or both, depending on the site of injury. Motor symptoms are divided according to those caused by lower motor neuron injury and those caused by upper motor neuron injury. In general, injury of the anterior horn leads to symptoms of lower motor neuron injury, whereas injury of the corticospinal tract presents with symptoms of upper motor neuron injury; however, it can be difficult to identify exact symptom etiology when two different lesions affect structures in the same location. For example, in cases involving both anterior horn disease and a peripheral neuronal lesion, it is difficult to distinguish whether or not lower motor neuron injury symp-

Manuscript accepted for publication November 29, 2016

aDepartment of Neurology, Eulji University Hospital, Eulji University School of Medicine, Dunsan Seo-ro 95, Seo-gu, Daejeon 35233, Korea

${ }^{b}$ Corresponding Author: Do-Hyung Kim, Department of Neurology, Eulji University Hospital, School of Medicine, Eulji University, Dunsan Seo-ro 95, Seo-gu, Daejeon 35233, Korea. Email: semoxx@naver.com

doi: https://doi.org/10.14740/jnr407w toms are related to anterior horn damage [1]. These cases necessitate meticulous clinical observation, neurological examination, electrophysiological testing, and neuroimaging. Here, we report the case of a patient who was initially diagnosed with a tibial neuropathy caused by a tumor affecting the first sacral nerve, and instead later diagnosed with likely anterior horn disease.

\section{Case Report}

A 23-year-old woman was admitted to our outpatient neurology clinic complaining of progressive right lower limb weakness that had onset 4 years prior. The patient said that she usually had trouble standing on the tips of her right toes, and that it was difficult to place weight on her right ankle when walking. While there were no other sensory symptoms, the patient had developed pes cavus about 3 years earlier; weakness had progressed to atrophy in her right leg and on the soles of her feet (Fig. 1), with discomfort upon plantar flexion of the right foot. She had no other notable history of medication use, trauma, exposure to hazardous materials, or family history. The patient underwent intravenous steroid therapy at a different hospital, but weakness and muscular atrophy were worsened by the therapy.

Upon admission, the patient did not exhibit any consciousness disturbance and had normal cranial nerve examination findings. Limb strength was normal in the upper extremities, lower left limb, right proximal lower extremity, and right knee. The dorsiflexor and evertor of the right ankle were also normal, but weakness in the plantar flexor, invertor, and toe plantar flexor was Medical Research Council (MRC) grade 4-4-. The patient had normal sensory function in all extremities, but the right ankle deep tendon reflex was absent.

Nerve conduction study (NCS) revealed decreased compound muscle action potential (CMAP) amplitudes in the right medial and lateral plantar nerves, as well as the right tibial nerve relative to the contralateral side. Moreover, F-wave latencies were prolonged in right leg compared to the contralateral side. Findings were normal for the right femoral, saphenous, peroneal, and plantar sensory nerves, the nerves of the left lower limb, the nerves of the bilateral upper limbs, and the left H-reflex latencies. Electromyography (EMG) indicated denervation and reduced interference patterns in the right abductor digiti minimi, abductor pollicis brevis, tibialis posterior, medial gastrocnemius, semimembranosus, and semitendinosus 


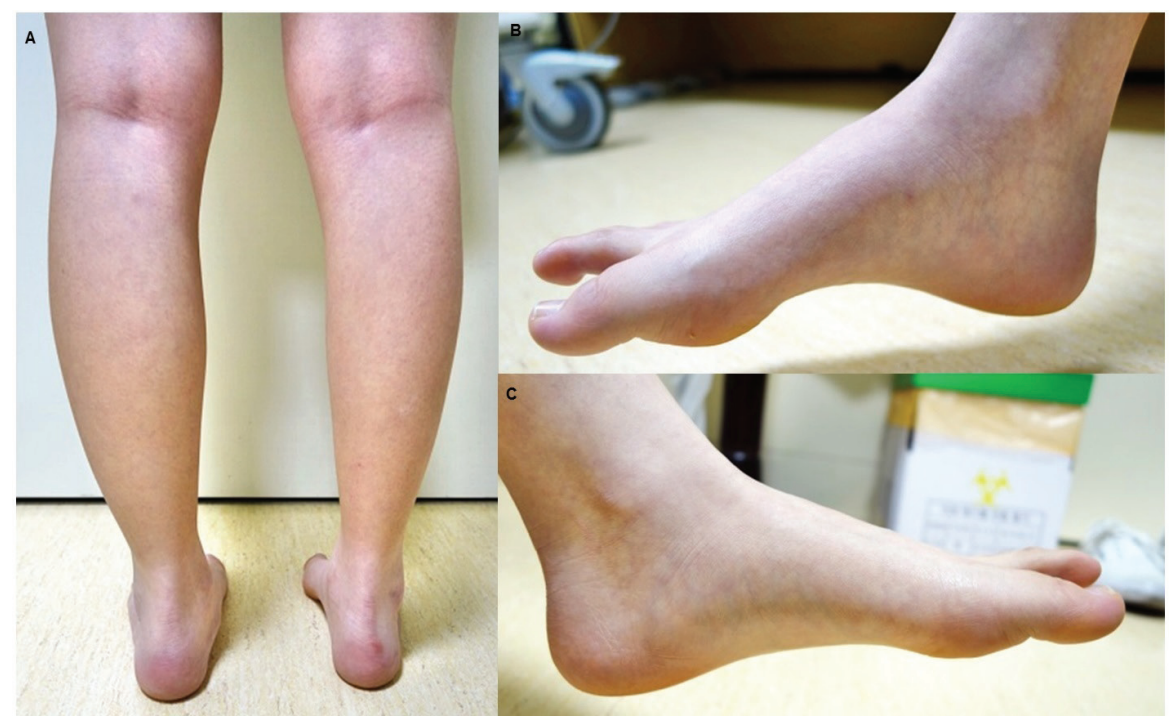

Figure 1. Photographs of lower limbs of patient show marked muscle atrophy of posterior compartment of right calf (A) and evidence of pes cavus (B). The left plantar muscle is normal (C).

muscles, as well as giant motor unit action potentials (MUAPs) in the semimembranosus, tibialis posterior, and medial gastrocnemius muscles. The remaining lower and upper limb muscles and paraspinal muscles were normal (Tables 1 and 2). Sensory-evoked potential testing results for the right and left upper and lower limbs were also normal. Neurological and electrophysiological testing suggested a lesion localized to the tibial nerve branch originating from the proximal area of the right sciatic nerve. Pelvic MRI showed overall atrophy and fat tissue deposition in the muscles of the deep posterior compartment, including the plantaris, gastrocnemius, and soleus muscles, as well as in the semimembranosus and semitendinosus (hamstring) muscles. In addition, diffuse swelling of the sciatic nerve was detected between the right piriform muscle and popliteal fossa. Further, we observed a mass originating in the middle of the sacral nerve plexus where the first sacral nerve passes through the pelvic sacral foramen and branches. Both the mass and diffuse swelling showed high-intensity signal on T2-weighted imaging, but no increases in signal intensity or contrast enhancement were observed on T1-weighted imaging (Fig. 2). Blood, urine, thyroid function, autoantibody, Charcot-Marie-Tooth disease, tumor marker, and cerebrospinal fluid test results were all normal, and no abnormalities were observed on whole spine MRI.

After diagnosing an intraneural ganglion of the right sacral nerve plexus, the patient was scheduled for surgery at the orthopedic center. However, during surgery, the mass was not observed on MRI and it was confirmed that the mass was in fact a hyperemia firmly entrapped by the surrounding tissues. The entrapped tissue and nerve were detached. Adjacent tissue and sheath biopsies were directly sampled and were normal.

About 11 months after surgery, the patient returned to the outpatient neurology center complaining of unchanged lower extremity weakness despite regular rehabilitation therapy after surgery. Indeed, plantar flexor, invertor, and toe plantar flexor were the same MRC grade, and right lower limb atrophy was unchanged. Once again, the patient did not present any sensory symptoms and did not exhibit atrophy of any other joints. Follow-up NCS and bilateral EMG results were largely unchanged relative to the first visit.

\section{Discussion}

Upon initial presentation, the patient in this case report was thought to have distally localized tibial motor neuropathy without sensory involvement. Electrophysiological results indicated a lesion of the tibial nerve branch of the right proximal sciatic nerve, and MRI revealed a suspected ganglion lesion affecting the first sacral nerve of the sacral nerve plexus. Intraoperatively, we found that the suspected ganglion was in fact an entrapment lesion, and detachment was performed. However, the patient's clinical symptoms and electrophysiological deficits were not improved after surgery and rehabilitation.

Entrapment neuropathy of the lower extremities is relatively common affecting the sciatic nerve near the piriformis muscle, the tibial nerve near the popliteal fossa and tarsal tunnel, the common peroneal nerve near the neck of the fibula, and the digital nerve near the head of the metatarsal bone [2]. Depending on the level of injury, entrapment can lead to various motor dysfunctions such as weakness, palsy, and atrophy, sensory dysfunctions such as dysesthesia, and autonomic dysfunctions such as anhidrosis and ulceration in the areas innervated by the entrapped nerve. In particular, pain and dysesthesia are the most common symptoms in the earliest phases of entrapment [3]. A previous case report of a tumor along the sacral nerve passing through the first left pelvic sacral foramen indicated the presence of clear sensory symptoms (pain worsened by movement), atrophy of the innervated muscles, and loss of sensory nerve action potentials in the left sural nerve [4]. The patient in our case had a lesion in a similar location that progressed to atrophy; however, our patient did not exhibit 
Table 1. Result of Nerve Conduction Study of Bilateral Lower Extremities

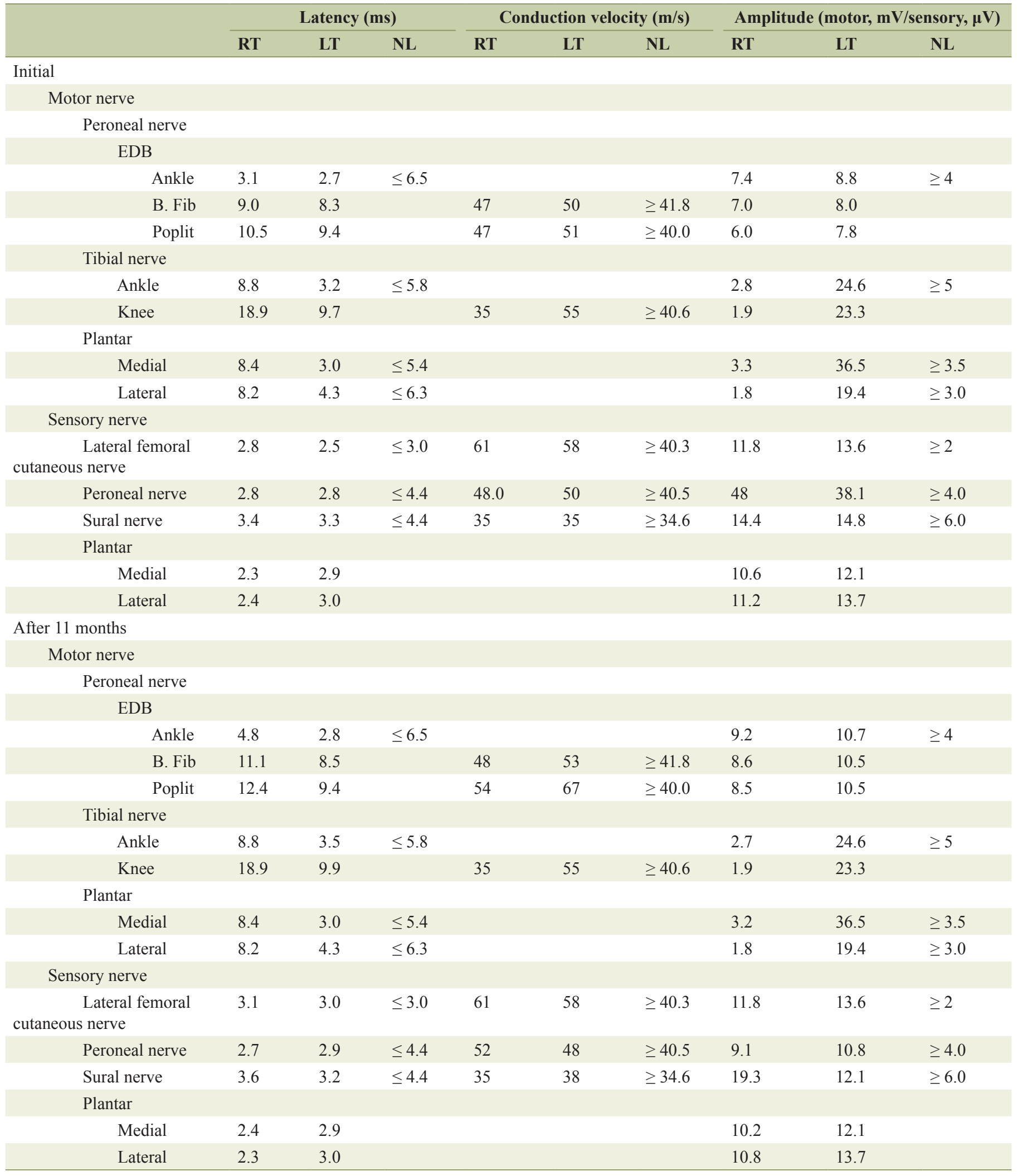

RT: right; LT: left; NL: normal range; NR: no response; EDB: extensor digitorum brevis; B. Fib: below fibular head; Poplit: popliteal fossa; Fib head: fibular head. 
Table 2. Result of Electromyography of Bilateral Lower Extremities

\begin{tabular}{|c|c|c|c|c|c|}
\hline Muscle & Insertional activity & Fibrillation & Positive sharp wave & MUAP & IP \\
\hline \multicolumn{6}{|c|}{ Gluteus maximus } \\
\hline RT & Normal & - & - & Normal & FIP \\
\hline \multicolumn{6}{|c|}{ Gluteus medius } \\
\hline RT & Normal & - & - & Normal & FIP \\
\hline \multicolumn{6}{|c|}{ Vastus lateralis } \\
\hline RT & Normal & - & - & Normal & FIP \\
\hline LT & Normal & - & - & Normal & FIP \\
\hline \multicolumn{6}{|c|}{ Biceps femoris short and long head } \\
\hline RT & $\uparrow$ & + & ++ & Increased & RIP \\
\hline LT & Normal & - & - & Normal & FIP \\
\hline \multicolumn{6}{|c|}{ Semitendinosus } \\
\hline RT & $\uparrow$ & - & ++ & Normal & RIP \\
\hline LT & Normal & - & - & Normal & FIP \\
\hline \multicolumn{6}{|c|}{ Tibialis anterior } \\
\hline RT & Normal & - & - & Normal & FIP \\
\hline LT & Normal & - & - & Normal & FIP \\
\hline \multicolumn{6}{|c|}{ Gastrocnemius medius } \\
\hline RT & $\uparrow$ & + & ++ & Increased & RIP \\
\hline LT & Normal & - & - & Normal & FIP \\
\hline \multicolumn{6}{|c|}{ Abductor halluces brevis } \\
\hline RT & $\uparrow$ & - & ++ & $\mathrm{NP}$ & - \\
\hline LT & Normal & - & - & Normal & FIP \\
\hline \multicolumn{6}{|c|}{ Abductor digiti quinti pedis } \\
\hline RT & $\uparrow$ & - & ++ & NP & - \\
\hline LT & Normal & - & - & Normal & FIP \\
\hline \multicolumn{6}{|c|}{ Paraspinal (C4-T1, L1-S2) } \\
\hline RT & Normal & - & - & - & - \\
\hline LT & Normal & - & - & - & - \\
\hline
\end{tabular}

RT: right; LT: left; MUAP: motor unit action potential; IP: interference pattern; NP: non-production of MUAP; RIP: reduced interference pattern; FIP: full interference pattern. 


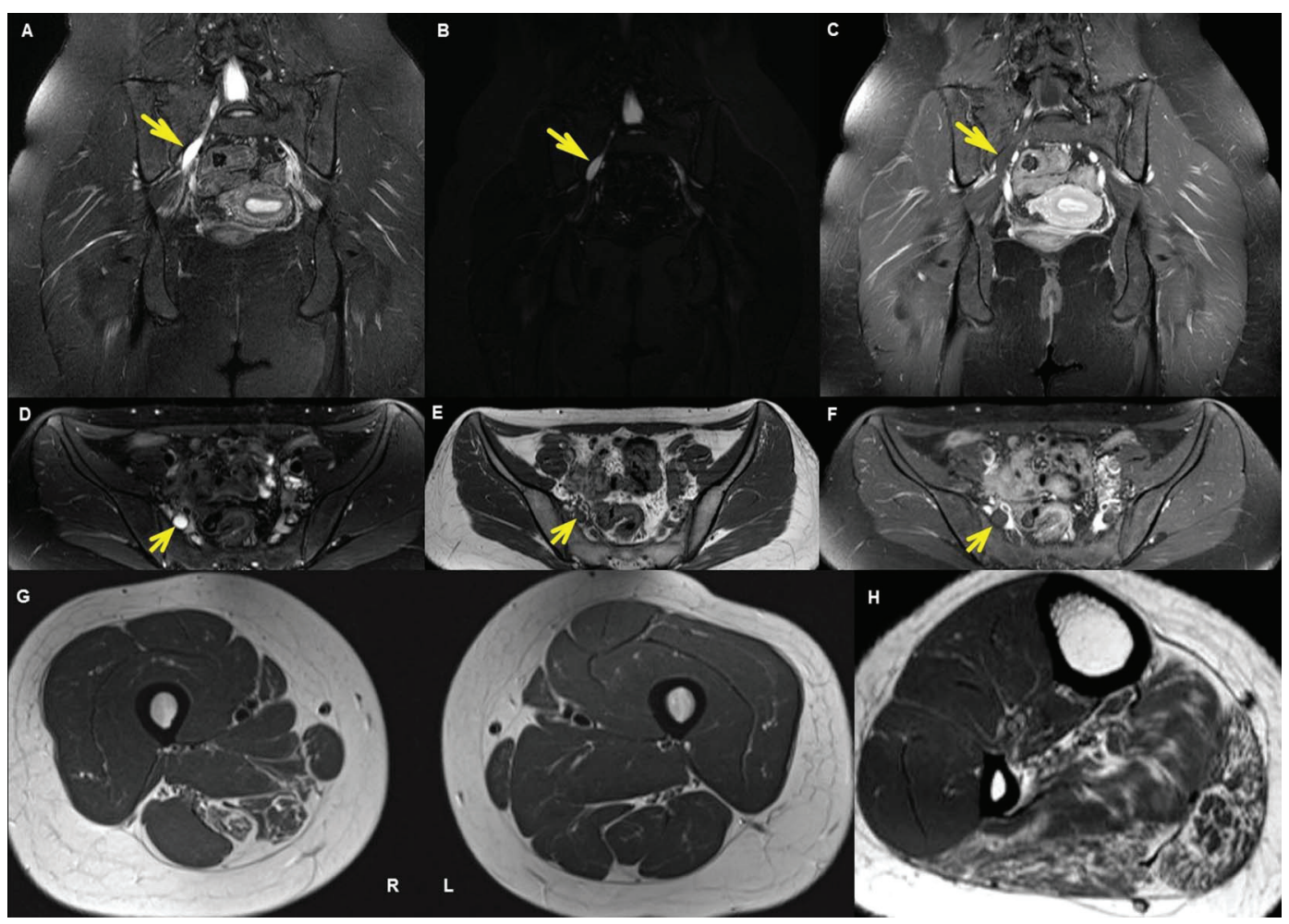

Figure 2. MRI of pelvis. (A), (B), (C) are coronal and (D), (E), (F) are axial images of the pelvis. Coronal (A) and axial (D) T2weighted images and coronal maximum intensity projection (MIP) sampling perfection with application optimized contrasts using varying flip angle evolutions (SPACE) image (B) shows a 2.2-cm diffuse nerve swelling probably representing a mass and increased signal intensity in right S1 spinal nerve as it courses out of the sacral foramen. The lesion of diffuse nerve swelling does not show enhancement on coronal (C) and axial (F) T1-gadolinium enhancement sequence. Fat-suppressed turbo-spin-echo (TSE) T1 axial image (E) shows low signal intensity along the right swelling lesion anterior to sacrum. Axial T1-weighted images (G) of the thigh shows fat replacement and atrophic change in right adductor magus, semimembranosus, and semitendinosus muscles and increased signal intensity. Axial T1-weighted image $(\mathrm{H})$ of the calf shows fat replacement and atrophic change in right gastrocnemius, soleus, plantaris and posterior compartment muscles.

sensory symptoms and had normal sural and plantar sensory nerve conduction. In general, peripheral nerve or nerve plexus disorders present with abnormal findings in sensory nerve conduction studies, while disorders involving lesions proximal to the dorsal root ganglion (e.g., myeloradiculopathy) show normal findings in sensory nerve conduction studies $[4,5]$. The fact that our patient had normal right sural and plantar sensory nerve conduction suggested that the lesion was proximal to the dorsal root ganglion, and the absence of sensory symptoms suggested that the patient's symptoms were associated with an etiology different from that of entrapment or a tumor affecting the first sacral nerve. Further, the fact that the patient did not show clinical or electrophysiological improvements after detachment of the nerve from the entrapping tissue suggested that her symptoms were irrelevant to the entrapment radiculopathy and spinal diseases are possible candidates explaining a lesion proximal to the dorsal root ganglion; however, considering that the lesion shown on whole spine MRI did not involve a spinal nerve root proximal to the dorsal root ganglion of the first sacral nerve, and given that the patient exhibited specific injury of the tibial motor nerve axons, spinal motor neuron disease was most likely.

Damage to the anterior horn cell produces symptoms of lower motor neuron injury, while damage to the corticospinal tract results in symptoms of upper motor neuron injury. The patient in this case showed weakness and atrophy of the muscles innervated by the tibial motor nerve, and a complete loss of the deep tendon reflex in the right ankle, presumably due to right-localized right motor neuron disease involving the cells of the first sacral nerve in the anterior horn. This pattern could represent a number of diseases including amyotrophic lateral sclerosis, spinal muscular atrophy, spinobulbar muscular atrophy, poliomyelitis, and monomelic amyotrophy [6]. Here, we suspected monomelic amyotrophy based on the fact that patient had no other forms of existing disease or family history, exhibited symptoms localized to the right lower limb, and had normal electrophysiological findings with regard to other lower and upper limbs and paraspinal muscles. Yet, because monomelic amyotrophy generally affects the upper extremities and frequently presents in male adolescents [7-9], additional observation is required before a definitive diagnosis can be made. Nerve compression induces edema within the endoneurium and thickening of the internal epineurium and perineurium, which also become enlarged due to microvascular proliferation. Although chronic compression thins the affected nerve, the area surrounding the site of compression is enlarged through this 
mechanism [10], and may have caused the observed ganglionlike lesion of the first sacral nerve shown on MRI in our case.

This case stresses that, if several diseases are suspected as the cause of a particular set of sensory or motor clinical symptoms, it is important to habituate the lesion localization and verify that results of multiple imaging tests or other testing modalities match the findings of neurological examinations.

\section{Acknowledgments}

The authors appreciate the patient for accepting publication of her clinical and radiological information.

\section{Conflicts of Interest}

The authors declare no conflicts of interest.

\section{References}

1. Marx A, Glass JD, Sutter RW. Differential diagnosis of acute flaccid paralysis and its role in poliomyelitis surveillance. Epidemiol Rev. 2000;22(2):298-316.

2. Kim S, Choi JY, Huh YM, Song HT, Lee SA, Kim SM, Suh JS. Role of magnetic resonance imaging in entrapment and compressive neuropathy - what, where, and how to see the peripheral nerves on the musculoskeletal magnetic resonance image: part 1. Overview and lower extremity. Eur Radiol. 2007;17(1):139-149.

3. Donovan A, Rosenberg ZS, Cavalcanti CF. MR imaging of entrapment neuropathies of the lower extremity. Part 2. The knee, leg, ankle, and foot. Radiographics. 2010;30(4):1001-1019.

4. Feinberg J, Sethi S. Sciatic neuropathy: case report and discussion of the literature on postoperative sciatic neuropathy and sciatic nerve tumors. HSS J. 2006;2(2):181187.

5. Simmons Z. Electrodiagnosis of brachial plexopathies and proximal upper extremity neuropathies. Phys Med Rehabil Clin N Am. 2013;24(1):13-32.

6. Tiryaki E, Horak HA. ALS and other motor neuron diseases. Continuum (Minneap Minn). 2014;20(5 Peripheral Nervous System Disorders):1185-1207.

7. Foster E, Tsang BK, Kam A, Storey E, Day B, Hill A. Hirayama disease. J Clin Neurosci. 2015;22(6):951-954.

8. Hashimoto M, Yoshioka M, Sakimoto Y, Suzuki M. A 20-year-old female with Hirayama disease complicated with dysplasia of the cervical vertebrae and degeneration of intervertebral discs. BMJ Case Rep. 2012;2012.

9. Schnyder H, Meyer M. [Benign, focal amyotrophy]. Schweiz Med Wochenschr. 1991;121(6):167-173.

10. Chung YG, Kim BG. Ultrasonographic examination of compression neuropathy in the upper extremity. J Korean Orthop US Soc. 2008;1(1):64-72. 